\title{
Percentage fat fraction in magnetic resonance imaging: upgrading the osteoporosis-detecting parameter
}

\author{
Rong Chang ${ }^{1}$, Xiaowen Ma ${ }^{1}$, Yonghong Jiang ${ }^{1}$, Dageng Huang ${ }^{2}$, Xiujin Chen², Ming Zhang ${ }^{3^{*}}$ and Dingjun Hao 2* $^{2^{*}}$
}

\begin{abstract}
Background: Osteoporosis (OP) is a systemic metabolic bone disorder identified as an essential health issue worldwide. Orthopedic imaging approaches were commonly used with some limitations. Thus, our study aimed to investigate the diagnostic value of magnetic resonance spectroscopy (1-H MRS) and m-Dixon-Quant in OP.

Methods: A total of 76 subjects were enrolled in the study and bone mineral density (BMD) was measured using quantitative computed tomography $(\mathrm{QCT})$. Then, the subjects were divided into three groups according to BMD: normal control group, osteopenia group and OP group. The following parameters were recorded for each patient: gender, age, height, body weight, waist circumference, and hip circumference. Further, the fat fraction percentage (FF\%) values were determined by 1-H MRS and m-Dixon-Quant methods.

Results: In both 1-H MRS and magnetic resonance Imaging (MRI) m-Dixon-Quant, the FF\% exhibited a negative correlation with BMD $(P<0.05)$. The FF\% value of the OP group was significantly higher than that of the control group $(P<0.05)$. In addition, the FF\% value in the m-Dixon scans was positively related to age, while BMD showed a negative linear relationship with age $(P<0.0001)$. Further, females had a significantly higher FF\% value compared to males $(P<0.01)$, and height was correlated with BMD $(P<0.05)$ but not with FF\% $(P>0.05)$.

Conclusions: MRI investigations especially FF\% value in the m-Dixon-Quant imaging system is correlated with OP. Its diagnostic value remains to be demonstrated on a large prospective cohort of patients. Besides, parameters such as age, gender, and height are important factors for predicting and diagnosing OP.
\end{abstract}

Keywords: Osteoporosis, Bone mineral density, Magnetic resonance spectroscopy, Fat fraction; MRI m-Dixon-quant

\section{Background}

Osteoporosis (OP) is a systemic metabolic bone disorder that affects more than 200 million people especially the elderly worldwide [1]. It is characterized by reduced bone density, leading to an increase in bone fragility and susceptibility to fractures, most commonly vertebral compression fracture (VCF) $[2,3]$. Previous study showed that disability rate of OP would be tripled by 2050 with a heavy economic and social burden for the family and society [4]. $\mathrm{OP}$ is associated with increased bone marrow adipocytes and impaired bone formation [5]. The formation of bone,

\footnotetext{
*Correspondence: zmmri@163.com; cr4512015105@163.com

${ }^{3}$ Department of Medical Imaging, First Affiliated Hospital, Medical College

Xi'an Jiaotong University, Xi'an 710061, China

${ }^{2}$ Department of Orthopedics, Honghui Hospital, Xi'an Jiaotong University

College of Medicine, Xi'an 710054, China

Full list of author information is available at the end of the article
}

hematopoiesis and osteoclast, regulated the signaling pathway in the process of bone marrow adipocyte formation, which is a potential molecular target for the prevention and treatment of OP.

Since the 1990s, the World Health Organization (WHO) has defined OP based on the BMD determined by dual-energy X-ray absorptiometry (DXA), which has been used worldwide [6]. The current detection of BMD is an important basis for the diagnosis of OP; however, it has some limitations since BMD only reflects a part of bone mass, but not the nature of bone marrow, minerals, organic matter and other ingredients [7]. Studies have shown that BMD is correctly diagnosed in only 10 to $53 \%$ of older postmenopausal women [8]. BMD evaluation by DXA also has several known limitations since patients were commonly misclassified as having false-

(c) The Author(s). 2020 Open Access This article is distributed under the terms of the Creative Commons Attribution 4.0 International License (http://creativecommons.org/licenses/by/4.0/), which permits unrestricted use, distribution, and 
negative diagnoses and subsequently received delayed treatments [9]. However, quantitative computed tomography (QCT) and MRS can be used to quantitatively or semi-quantitatively analyze bone micro-structure and intramedullary lipid of OP. And the changes of bone microstructure and bone marrow biomolecular components can be detected before abnormal BMD. Combined application can comprehensively, quantitatively and noninvasively evaluate the bone quality and predict the bone strength, showing a broad application prospect in the OP diagnosis and efficacy monitoring [10]. Nevertheless, QCT has ionizing radiation and it is limited in differentiating healed from non-healed fractures [11].

Therefore, novel tools were urgently needed to diagnose $\mathrm{OP}$, assess fracture risk, and monitor response to therapy. Magnetic resonance imaging (MRI) is a useful noninvasive tool for acquiring in vivo images without $\mathrm{X}$-ray ionizing radiation. It has been revealed that lumbar spine MRI routinely performed for low back pain can be used as an opportunistic screening tool for OP without additional cost, radiation exposure or patients time, but it needs DXA as a reference standard [12]. MR spectroscopy (MRS) belongs to one of the most useful approaches in MRI, which can differentiate healed from non-healed fractures in the human body [13]. Generally, it is expressed by detecting the resonance frequency between the substance and the reference to obtain a relative value of parts per million, which has been gradually applied to the diagnosis of various diseases including tumors [14]. Previous study of the lumbar spine has indicated that bone density has a negative relationship with bone marrow fat content [15]. The $1 \mathrm{H}$ MRS method is the only noninvasive method providing information on the biochemical profile of patient tissues in vivo [16]. Further, the m-Dixon-Quant technique is a novel development of MRI [17]. It arbitrarily selects echoes in the signal-acquisition process, thus effectively shortening the TE time. The m-Dixon-Quant technique combines parallel acquisition technologies such as SENSE and dS-SENSE to enhance the imaging speed, and greatly improves the resolution with minimal noise ratio. This technique was found to be effective at calculating lipid content in vivo [18].

One known mechanism of spinal OP is that the adipogenic differentiation of mesenchymal stem cells (MSCs) is upregulated compared to osteogenic differentiation in spinal marrow. The fat fraction (FF) would increase when this balance is broken, which can be assessed using MRS and m-Dixon-Quant. Thus, we hypothesized that the FF percentage (FF\%) could be used to diagnose OP. In order to investigate the diagnostic value of 1-H MRS and m-Dixon-Quant in the evaluation of OP, we combined results of the two methods and proposed a novel diagnostic strategy for clinically quantitative analysis of OP.

\section{Methods}

\section{Patients}

This study was approved by the Ethics Committee of Hong Hui Hospital, Xi'an Jiaotong University, and performed between May 2016 and October 2017. A total of 76 subjects with mean age of $59.18 \pm 9.22$ years were enrolled, 46 males (mean age $59.17 \pm 9.06$ years) and 30 females (mean age $59.20 \pm 9.61$ years) were enrolled in the study. All the subjects had no vertebral fractures previously and were divided into three groups according to the results of BMD measurement: normal control group (18 cases), osteopenia group (30 cases) and OP group (28 cases). The inclusion criteria were as follows: (1) clinical data were complete, routine MRI scan and $1 \mathrm{H}$ MRS test were performed; (2) informed consent was signed. The exclusion criteria were as follows: (1) clinical data were incomplete; (2) patients with tumors (3) with other metabolic and endocrine diseases. The following parameters were recorded for each patient: gender, age, height, body weight, waist circumference, and hip circumference. All patients signed informed consent and agreed to participate in the survey.

\section{BMD measurements}

QCT PRO software version 5.1 and Mindways were used for the BMD measurements with synchronous calibration. All the patients underwent QCT scanning of the anterior-posterior lumbar spine (L2-L4) and their BMD values were expressed in $\mathrm{mg} / \mathrm{cm}^{3}$. PHILIPS 16-slice spiral CT reconstruction was applied to obtain QCT values.

According to the American College of Radiology Guidelines for QCT, all the subjects were divided into three categories based on their BMD results: normal control group $\left(>120 \mathrm{mg} / \mathrm{cm}^{3}\right)$, osteopenia group $(80$ $\left.120 \mathrm{mg} / \mathrm{cm}^{3}\right)$ and OP group $\left(<80 \mathrm{mg} / \mathrm{cm}^{3}\right)$ [19].

The BMD values of the $\mathrm{L} 2, \mathrm{~L} 3$, and $\mathrm{L} 4$ vertebrae were obtained, and the average BMD was calculated using the mean value of L2, L3, and L4. An OP or osteopenia diagnosis was primarily determined in accordance with the average $B M D$ value.

\section{1-H MRS examination}

Routine MR scans and 1-H MRS scans were performed in all subjects when available. In all cases, 52 cases received the MR scan. The $1.5 \mathrm{~T}$ PHILIPS superconducting MR machine (Philips Medical Systems, Best, Netherlands) was used for spectral acquisition. Through conventional MRI plain scanning, as well as sagittal and transverse scanning, the following indexes were set: T2WI/TSE TR 2500, TE 100, two acquisitions, FOV 160*302*57 mm, layer thickness $4 \mathrm{~mm}$, spacing $0.8 \mathrm{~mm}$, matrix 180*237; and T1WI/TSE TR 400, TE 8, FOV $160 * 299 * 57 \mathrm{~mm}$, layer thickness $4 \mathrm{~mm}$, spacing $0.8 \mathrm{~mm}$, matrix $160 * 214$. The $1 \mathrm{H}$ MRS scan used a single element 
Point Resolved Spectroscopy (PRESS) sequences for the sagittal and transverse scans. The MRS scan parameters were as follows: TR $2000 \mathrm{~ms}$; TE $42 \mathrm{~ms}$; wave width $1000 \mathrm{~ms}$; excitation frequency 120 times; voxel 15 $\mathrm{mm} * 15 \mathrm{~mm} * 12 \mathrm{~mm}$. The area was reproducible and easy to operate. Thereby, the middle vertebral body was taken as the representative. Based on the previous research [20], we recorded the water peak of the L3 vertebral body displacement around $4.70 \mathrm{ppm}$ and the fat peak between 1.30 and $0.90 \mathrm{ppm}$.

The FF\% values were calculated using the formula: $\mathrm{FF} \%=\mathrm{I}_{\text {fat }} /\left(\mathrm{I}_{\text {water }}+\mathrm{I}_{\mathrm{fat}}\right) * 100 \%$, where $\mathrm{I}_{\text {water }}$ or $\mathrm{I}_{\text {fat }}$ represented the peak of fat or water in the examined substance [21]. This parameter refers to the relative fat signal strength amplitude in relation to the total amplitude (water + fat). After scanning with 1H MRS and m-DixonQuant sequences, post-processing software of PHILIPS MR machine automatically calculated $\mathrm{I}_{\text {water }}$ or $\mathrm{I}_{\text {fat }}$ values.

\section{M-Dixon-quant scanning}

Meanwhile, the $1.5 \mathrm{~T}$ PHILIPS superconducting MR machine (Philips Medical Systems, Best, Netherlands) was used to capture the m-Dixon-Quant images. The conventional settings were identical to the $1 \mathrm{H}$ MRS protocols. For the MRI m-Dixon-Quant 3D scan, six phase diagrams were acquired toward three lumbar vertebrae. The medullary cavity was in the central region of the vertebral body, avoiding the cortical bone at the edge of the vertebral body, and a rectangle of $15 \mathrm{~mm}^{*} 15 \mathrm{~mm}^{*} 12 \mathrm{~mm}$ was drawn at the center of the L3 vertebral body as the region of interest. Then, we measured on the fat map made by $1 \mathrm{~m}$-Dixon-Quant. The scanning parameters were as follows: TR $7 \mathrm{~ms}$; multi-echo; echo chain 6; NSA 2, flip angle 5\%; FOV 300*369*120; voxel
$2 * 2 * 4$; layer thickness $6 \mathrm{~mm}$; spacing $3 \mathrm{~mm}$; matrix $152 * 184 * 60$; scan mode 3D; and scan time $51 \mathrm{~s}$. The FF\% value was directly reported by the PHILIPS superconducting MR machine (with the Philips 3.0tx superconducting MRI scanner software). The target area of the MRI analysis was the same as the area of the BMD measurement point.

\section{Statistical analysis}

All results were presented as mean \pm standard deviation (SD). The relationship between pairs of variables was assessed using the linear correlation analysis (Pearson's $\mathrm{R}$ coefficient). Differences between groups were assessed by one-way ANOVA and the difference between males and females was compared using unpaired $t$-tests. $P$ values $<0.05$ were considered statistically significant.

\section{Results}

FF is negatively correlated with bone density

First, the parameter FF\% was used in both $1 \mathrm{H}$ MRS and m-Dixon-Quant methods, and the results showed that the average BMD had a negative relationship with $\mathrm{FF} \%$ value. As shown in Fig. 1a, the 1H MRS data included 52 cases and a significant non-zero slope was found $\left(\mathrm{Y}=-0.1906 * \mathrm{X}+75.08 ; \quad\right.$ Pearson's $\quad R^{2}=0.1046 ; \quad P=$ 0.0194). Similarly, data in $m$-Dixon-Quant imaging included 76 cases showed a significantly negative correlation between the average bone density and FF\% (Y = $0.1201 * \mathrm{X}+69.15$; Pearson's $\left.R^{2}=0.2200 ; P<0.001\right)$. The patients were divided into three groups, and a consistent conclusion was drawn (Fig. 1b). The FF\% value of the OP group was significantly higher than that of the normal control group (m-Dixon-Quant method: FF\%= $51.25 \pm 7.38$ in the control group; $54.70 \pm 8.30$ in the

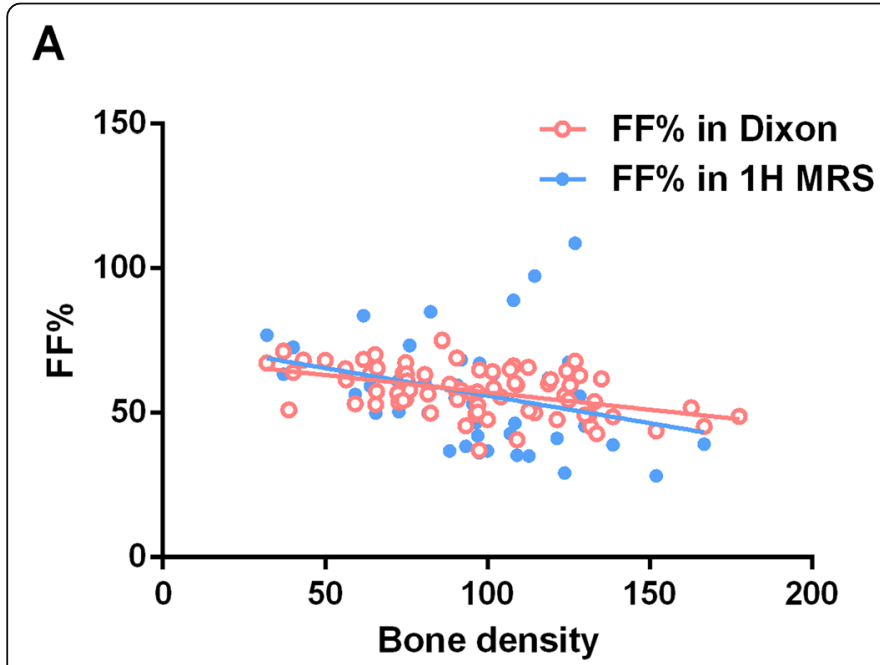

\section{B}

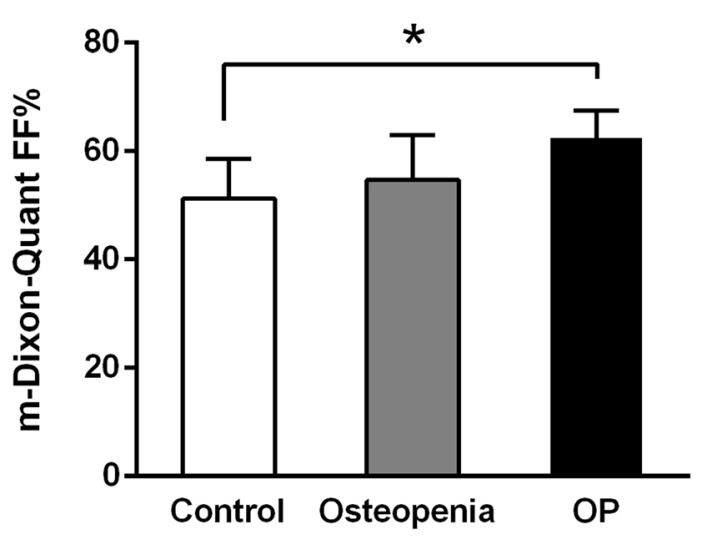

Fig. 1 FF is negatively correlated with BMD. a The FF\% for both $1 \mathrm{H}$ MRS and m-Dixon-Quant imaging had a significantly negative correlation with the average bone density value. $\mathbf{b}$ The patients were divided into three groups: normal control group, osteopenia group, and OP group; the FF\% value in the m-Dixon-Quant method was significantly higher in the OP group compared to normal control group. The bar presents the standard deviation; * $P<0.05$ 
osteopenia group; and $62.53 \pm 5.02$ in the OP group; OP versus control, $P<0.05)$. Two individuals in the $\mathrm{OP}$ and control groups were presented in Fig. 2. The lipid peaks of the L2 vertebral body in OP patients was significantly increased compared with those in control group (Fig. $2 \mathrm{a}-\mathrm{b})$ in the $1 \mathrm{H}$ MRS quantitative analysis. The same trend was observed for the L3 vertebral body during the m-Dixon-Quant analysis (Fig. 2c-d).

\section{Gender and age correlate with BMD and FF\% value}

In addition to $\mathrm{FF} \%$, other variables such as the gender, age, height, and weight of each patient were analyzed to probe for more references that may be helpful in the diagnosis. The result showed that BMD and FF\% value were largely determined by age (Fig. 3a). The FF\% from the m-Dixon scan exhibited a positive correlation with age $\left(\mathrm{F}_{1,74}=16.35\right.$, $P<0.0001, \quad \mathrm{Y}=0.3807^{*} \mathrm{X}+34.97$, Pearson's $R^{2}=0.1810$ ), while BMD showed a negative linear relationship (F1, 74= 42.77, $P<0.0001, \quad \mathrm{Y}=-2.216 * \mathrm{X}+227.6$, Pearson's $R^{2}=$ $0.3727)$. This is consistent with the possibility that older adults are at greater risk for developing OP. Further, we found that females had a higher FF\% value than males ( $t$ test; $P<0.01$ ) (Fig. 3b). Interestingly, height was found to be positively related to BMD (F1, 72=4.747, $P<0.05, \mathrm{Y}=$ $0.9652 * \mathrm{X}-63.63$, Pearson's $\left.R^{2}=0.06185\right)$, but not FF\% ( $P=$ 0.064) (Fig. 3c). This finding was reasonable since higher height implied stronger bone density, but the correlation was far below the parameters of gender and age. Further, body weight, waist circumference, and hip circumference were not associated with BMD or FF\%. Hence, gender and age correlate with $\mathrm{BMD}$ and $\mathrm{FF} \%$ value, and these parameters may help to diagnose and predict OP when combined with $1 \mathrm{H}$ MRS or m-Dixon techniques.

\section{Discussion}

In this study, we applied $1 \mathrm{H}$ MRS and m-Dixon-Quant 3D scanning in diagnosing OP and proposed that $\mathrm{FF} \%$ may reflect the $\mathrm{BMD}$ value. We found that both $1 \mathrm{H}$ MRS and m-Dixon-Quant imaging could measure bone marrow fat content, which had high clinical value in the diagnosis of OP. Gender, age and height are also associated with OP.

MRI is the only non-invasive method to provide quantitative analysis of tissue metabolism, biochemical environments, and compounds in vivo. Researchers have revealed the relationship between BMD and MRI indexes such as MRI signal intensities and marrow fat in the evaluation of OP [22-24]. However, few studies directly use MRI, especially m-Dixon-Quant 3D scanning, to examine the changes in the L2-4 vertebral body and diagnosis of OP. The m-Dixon-Quant sequence has a short scan time of only $51 \mathrm{~s}$. During the period, the fat score map is intuitively displayed, and the lipid content is quickly quantified without recalculation. However, the m-Dixon-Quant sequence is not configured on all models with the latest development sequence, which is not fully used. We proposed that FF\% played a crucial role in MRI-based OP assessments and our results provided a potentially novel strategy for preventing and treating OP that extends beyond BMD observation. Besides, it has been indicated that whether BMD is of predictive value is still controversial. MRI results are correlated with BMD values and provide the early detection of trabecular lesions, fractures, and deformities of the spine [25], while QCT and MRI can image and quantify the three-dimensional structure of trabecular bone [26], hence the combination of these methods may

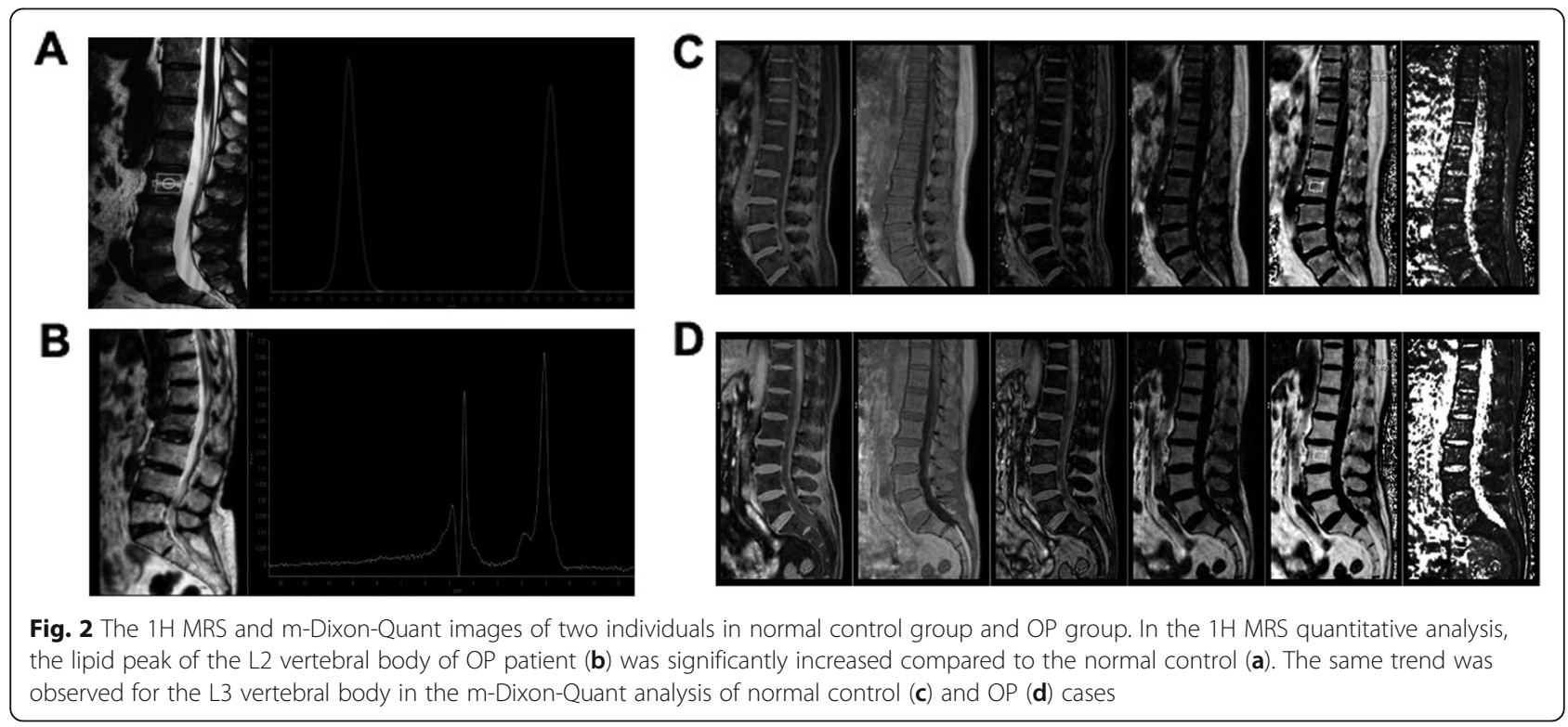



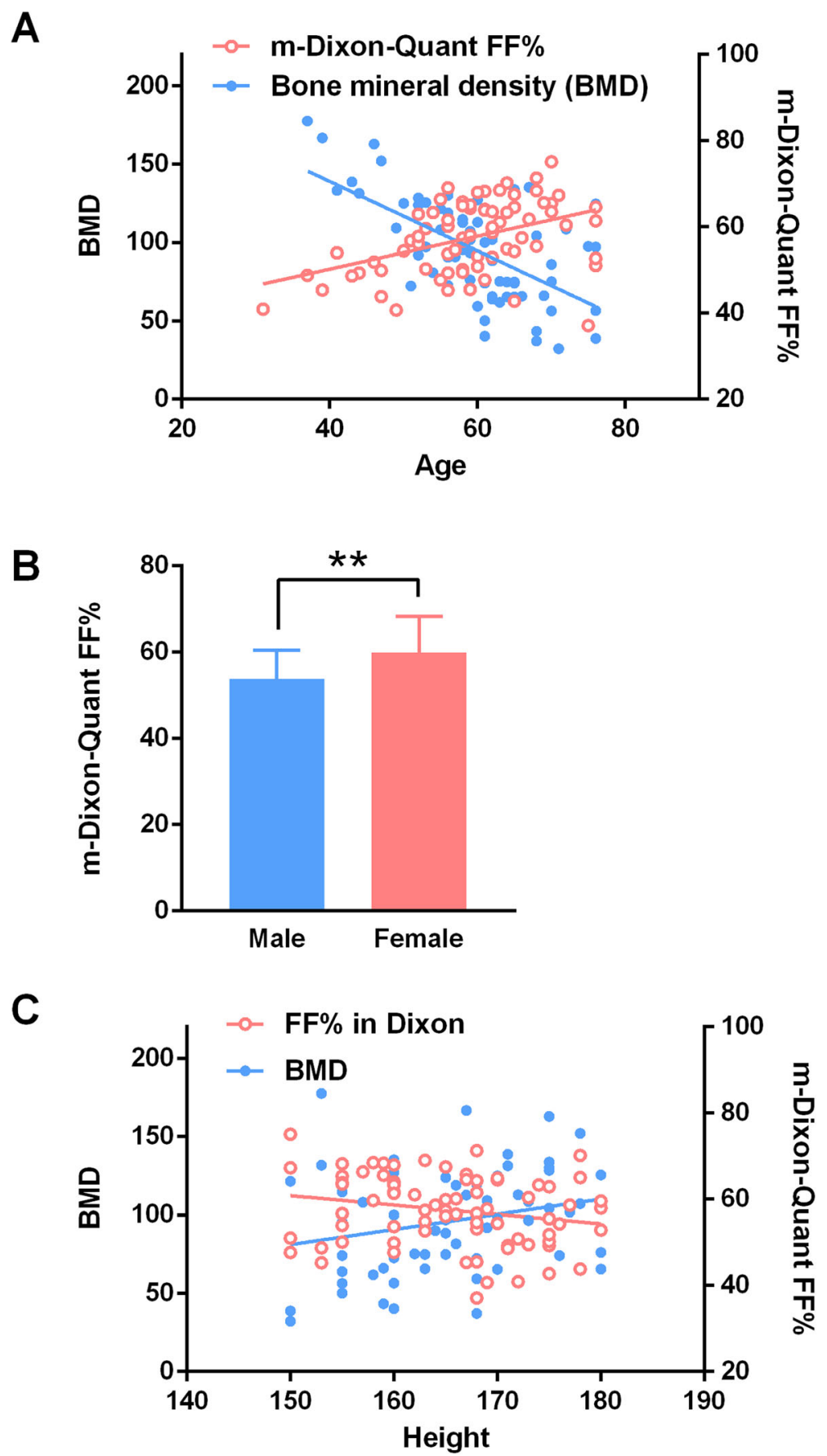

Fig. 3 Age, gender, and height correlated with BMD and FF\% values. a Age largely determined BMD and FF levels a. FF\% in m-Dixon exhibited a positive correlation with age, while BMD showed a negative linear relationship. $\mathbf{b}$ Females had a higher FF\% level compared to males. The bar presents the standard deviation; ${ }^{*} P<0.01$. c Height was correlated with BMD, but not FF\% $(P=0.064)$

be potential for prevention and treatment strategies. Our results suggest that $\mathrm{FF} \%$ and $\mathrm{BMD}$ permit the structural and metabolic status of vertebrae.

Nevertheless, a team from Italy used MRS and diffusion-weighted MRI to observe heels in normal controls, patients with osteopenia and $\mathrm{OP}$, and found no significant difference in marrow fat content between groups, while the effective internal magnetic field gradient (IMFG), a new MR parameter, held value in OP assessment [27]. This inconsistency with our result may be due to different patient profiles and bone tissues evaluated in both studies. 
Bone mass can be estimated by measuring FF using $1-\mathrm{H}$ MRS method. Our study showed that the FF\% is positively correlated with age, which is consistent with previous study [28]. The result may due to that the red bone marrow (RBM) changes physiologically to the yellow bone marrow (YBM), resulting in a relative decrease in water content in the red bone marrow and a relative increase in fat content [29]. Our study also found that women are more vulnerable to OP than men, since it has high incidence in postmenopausal women.

We also found that FF had a highly negative correlation with BMD. For patients with OP, RBM gradually transforms into YBM, causing a large number of fat cells to fill in the trabecular bone gap and the loss of dense bone material [30].

In this study, there was a significant difference of FF among the three groups. Through ROC analysis, sensitivity and specificity were high by using FF index to diagnose OP, which was up to $100 \%$ in the normal and OP group. FF can be used to diagnose OP since the bone fat content in OP patients is variable, and can reflect the pathological and physiological changes of OP [31].

However, DXA which represents the reference standard was not performed on these patients. Besides, QCT has an increasing fat error with increasing age and the number of cases in this study was small, increasing number of cases were needed to provide further diagnostic information for OP.

\section{Conclusions}

In conclusion, our study indicates that FF\% is related to age and BMD. The diagnosis of OP by $\mathrm{FF} \%$ has high sensitivity and specificity. The application of $1 \mathrm{H}$ MRS technology provides a new direction for evaluating and preventing osteoporosis but its diagnostic value remains to be demonstrated on a large prospective cohort of patients.

\section{Abbreviations}

OP: Osteoporosis; VCF: Vertebral compression fracture

\section{Acknowledgements}

Not applicable.

\section{Authors' contributions}

$\mathrm{RC}, \mathrm{XWM}$ and $\mathrm{YHJ}$ designed and analyzed the experiment, and was a major contributor in writing the manuscript. DGH, XJC, MZ and DJH performed the experiment. All authors read and approved the final manuscript.

\section{Funding}

This study was funded by a grant from the Development Center for Medical Science and Technology National Natural Science Foundation of China (NSFC) (No. 81772357) and Natural Science Foundation for Shaanxi of China (No. 2017JM8152).

These funds provide financial support for the article. We do research and articles based on the research ideas of the fund.

\section{Availability of data and materials}

All data generated or analyzed during this study are included in this published article [and its supplementary information files].

\section{Ethics approval and consent to participate}

The ethics committee of Honghui Hospital, Xi'an Jiaotong University College of Medicine approved the study.

\section{Consent for publication}

All contributing authors have agreed to submit this manuscript and all authors approved to publish this study.

We have obtained consent to publish from the participant to report individual patient data.

This study was written informed consent from the patients.

\section{Competing interests}

The authors declare that they have no competing interests.

\section{Author details}

'Department of Medical Imaging, Honghui Hospital, Xi'an Jiaotong University College of Medicine, Xi'an 710054, China. ${ }^{2}$ Department of Orthopedics, Honghui Hospital, Xi'an Jiaotong University College of Medicine, Xi'an 710054, China. ${ }^{3}$ Department of Medical Imaging, First Affiliated Hospital, Medical College Xi'an Jiaotong University, Xi'an 710061, China.

Received: 28 November 2019 Accepted: 10 February 2020

Published online: 17 March 2020

\section{References}

1. Karahan AY, Kaya B, Kuran B, Altındag O, Yildirim P, Dogan SC, Basaran A, Salbas E, Altınbilek T, Guler T. Common mistakes in the dual-energy X-ray absorptiometry (DXA) in Turkey. A retrospective descriptive multicenter study. Acta Med Austriaca. 2017;59(4):117-23.

2. Jarraya M, Hayashi D, Griffith JF, Guermazi A, Genant HK. Identification of Vertebral Fractures; 2013.

3. Chao CT, Chiang CK, Huang JW, Chan DC, group COoGNiNTUHs. Effect of frail phenotype on bone mass and vertebral compression fracture in individuals undergoing dialysis. J Am Geriatr Soc. 2016;64(9):e19-21.

4. Bouxsein ML, Seeman E. Quantifying the material and structural determinants of bone strength. 23(6):741-53.

5. Justesen J, Stenderup K, Ebbesen EN, Mosekilde LI, Kassem M. Adipocyte tissue volume in bone marrow is increased with aging and in patients with osteoporosis. Biogerontology. 2001;2(3):165-71.

6. Licks R, Licks V, Ourique F, Radke BH, Fontanella V. Development of a prediction tool for low bone mass based on clinical data and periapical radiography; 2014

7. Szathmári M. Evaluation of fracture risk in osteoporosis. Orv Hetil. 152(33): 1304-11.

8. Siris ES, Brenneman SK, Miller PD, Barrett-Connor E, Chen Y-T, Sherwood LM, Abbott TA. Predictive Value of Low BMD for 1-Year Fracture Outcomes Is Similar for Postmenopausal Women Ages 50-64 and 65 and Older: Results From the National Osteoporosis Risk Assessment (NORA). J Bone Miner Res. 19(8):1215-20.

9. Wainwright SA, Marshall LM, Ensrud KE, Cauley JA, Black DM, Hillier TA, Hochberg MC, Vogt MT, Orwoll ES. Study of osteoporotic fractures research G: hip fracture in women without osteoporosis. J Clin Endocrinol Metab. 2005;90(5):2787-93.

10. Shang W, Union P, Academy C, Union P. Beijing, China: Potential value of vertebral proton MR spectroscopy in determining osteoporosis. Chinese J Radiol. 2007.

11. Li X, Na L, Cheng X. Update on the Clinical Application of Quantitative Computed Tomography (QCT) in Osteoporosis. Current Radiology Reports. 2(10):1-5.

12. A new diagnostic score to detect osteoporosis in patients undergoing lumbar spine MRI. Eur Radiol. 25(10):2951-9.

13. Karchevsky M, Babb JS, Schweitzer ME. Can diffusion-weighted imaging be used to differentiate benign from pathologic fractures? A meta-analysis. Skeletal Radiol. 2008;37(9):791-5.

14. Wang CK, Li CW, Hsieh TJ, Chien SH, Tsai KB. Characterization of bone and soft-tissue tumors with in vivo $1 \mathrm{H}$ MR spectroscopy: initial Results1. Radiology. 2004;232(2):599-605. 
15. Ma HT, Rong R, Yang C, Griffith JF, Leung PC, Pu Z. A simulation study of marrow fat effect on bone biomechanics. 2014;2015(3):4030-3.

16. O'Brien CM, Vargis E, Paria BC, Bennett KA, Mahadevan-Jansen A, Reese J. Raman spectroscopy provides a noninvasive approach for determining biochemical composition of the pregnant cervix in vivo. Acta Paediatr. 2014; 103(7):715-21.

17. Hollak C, Maas M, Akkerman E, Den HA, Aerts H. Dixon quantitative chemical shift imaging is a sensitive tool for the evaluation of bone marrow responses to individualized doses of enzyme supplementation therapy in type 1 Gaucher disease. Blood Cells Mol Dis. 2001;27(6):1005-12.

18. Leo GD, Bandirali M, Fina L, Messina C, Sardanelli F. Measurement of vertebral bone marrow lipid profile at 1.5-T proton magnetic resonance spectroscopy and bone mineral density at dual-energy X-ray absorptiometry: correlation in a swine model. Skeletal Radiol. 43(8):1123-8.

19. Boos J, Brook A, Chingkoe CM, Morrison T, Brook OR. MDCT vs. MRI for incidental pancreatic cysts: measurement variability and impact on clinical management. Abdominal Radiol. 2016:42(2):1-10.

20. Dixon TW. Simple proton spectroscopic imaging. Radiology. 153(1):189-94.

21. Yeung DKW, Griffith JF, Antonio GE, Lee FKH, Woo J, Leung PC. Osteoporosis is associated with increased marrow fat content and decreased marrow fat unsaturation: A proton MR spectroscopy study. J Magn Reson Imaging. 22(2):279-85.

22. Hong J, Hipp JA, Mulkern RV, Jaramillo D, Snyder BD. Magnetic resonance imaging measurements of bone density and cross-sectional geometry. Calcif Tissue Int. 2000;66(1):74-8.

23. Ho KY, Hu HH, Keyak JH, Colletti PM, Powers CM. Measuring bone mineral density with fat-water MRI: comparison with computed tomography. J Magn Reson Imaging. 2012;37(1):237-42.

24. Patel PV, Eckstein F, Carballido-Gamio J, Phan C, Matsuura M, Lochmüller EM, Majumdar S, Link TM. Fuzzy Logic Structure Analysis of Trabecular Bone of the Calcaneus to Estimate Proximal Femur Fracture Load and Discriminate Subjects with and without Vertebral Fractures using HighResolution Magnetic Resonance Imaging at 1.5 T and $3 \mathrm{~T}$. Calcif Tissue Int. 81(4):294-304.

25. Becker C, Baltzer AW, Schneppenheim M, Becker A, Assheuer J, Merk HR, Krauspe R. Experimental validation of DXA and MRI-based bone density measurement by ash-method. Zentralbl Chir. 2001;126(5):402-6.

26. Ishida Y, Kawai S. Diagnostic imaging in osteoporosis (CT and MRI). Clin Calcium. 2001;11(12):1561-7.

27. Rebuzzi M, Vinicola V, Taggi F, Sabatini U, Wehrli FW, Capuani S. Potential diagnostic role of the MRI-derived internal magnetic field gradient in calcaneus cancellous bone for evaluating postmenopausal osteoporosis at 3T. Bone. 2013;57(1):155-63.

28. Fanucci E, Manenti G, Masala S, Laviani F, Costanzo GD, Ludovici A, Cozzolino V, Floris R, Simonetti G. Multiparameter characterisation of vertebral osteoporosis with 3-T MR. 112(2):208-23.

29. Wáng Y-XJ, Griffith JF, Deng M, Yeung DK, Yuan J. Rapid Increase in Marrow Fat Content and Decrease in Marrow Perfusion in Lumbar Vertebra Following Bilateral Oophorectomy: An MR Imaging-Based Prospective Longitudinal Study. Korean J Radiol. 16(1).

30. Ueda Y, Inaba M, Takada K, Fukui J, Sakaguchi Y, Tsuda M, Omae M, Kushida T, lida H, Ikehara S. Induction of Senile Osteoporosis in Normal Mice by Intra-Bone Marrow-Bone Marrow Transplantation from Osteoporosis-Prone Mice. Stem Cells (Miamisburg). 25(6):1356-63.

31. Zhang L, Li S, Hao S, Yuan Z. Quantification of fat deposition in bone marrow in the lumbar vertebra by proton MRS and in-phase and out-of-phase MRI for the diagnosis of osteoporosis. J X-Ray Sci Technol. 24(2):257-66.

\section{Publisher's Note}

Springer Nature remains neutral with regard to jurisdictional claims in published maps and institutional affiliations.

Ready to submit your research? Choose BMC and benefit from:

- fast, convenient online submission

- thorough peer review by experienced researchers in your field

- rapid publication on acceptance

- support for research data, including large and complex data types

- gold Open Access which fosters wider collaboration and increased citations

- maximum visibility for your research: over $100 \mathrm{M}$ website views per year

At BMC, research is always in progress.

Learn more biomedcentral.com/submissions 